\title{
Dynamic Changes in Brain Activity during Prism Adaptation
}

\author{
Jacques Luauté, ${ }^{1,2,3}$ Sophie Schwartz, ${ }^{3}$ Yves Rossetti, ${ }^{1,2}$ Mona Spiridon, ${ }^{3}$ Gilles Rode, ${ }^{1,2}$ Dominique Boisson, ${ }^{1,2}$ and \\ Patrik Vuilleumier ${ }^{3}$ \\ ${ }^{1}$ Université de Lyon, Université Lyon 1, Inserm UMR-S 864, 69500 Bron, France, ${ }^{2}$ Hospices Civils de Lyon, Service de Rééducation Neurologique, \\ Plateforme 'Mouvement et Handicap', Hôpital Henry Gabrielle, 69230 Saint-Genis-Laval, France, and '3aboratoire de Neurologie du Comportement et \\ Neuro-Imagerie Cognitive, Département de Neuroscience, Centre Médical Universitaire, CH-1211 Geneva, Switzerland
}

Prism adaptation does not only induce short-term sensorimotor plasticity, but also longer-term reorganization in the neural representation of space. We used event-related fMRI to study dynamic changes in brain activity during both early and prolonged exposure to visual prisms. Participants performed a pointing task before, during, and after prism exposure. Measures of trial-by-trial pointing errors and corrections allowed parametric analyses of brain activity as a function of performance. We show that during the earliest phase of prism exposure, anterior intraparietal sulcus was primarily implicated in error detection, whereas parieto-occipital sulcus was implicated in error correction. Cerebellum activity showed progressive increases during prism exposure, in accordance with a key role for spatial realignment. This time course further suggests that the cerebellum might promote neural changes in superior temporal cortex, which was selectively activated during the later phase of prism exposure and could mediate the effects of prism adaptation on cognitive spatial representations.

Key words: plasticity; fMRI; adaptation; cerebellum; spatial cognition; parietal

\section{Introduction}

Sensorimotor plasticity can be induced experimentally in healthy subjects using optical prisms that produce a misalignment between the perceived location of visual and proprioceptive information. Under prisms, initial pointing movements are typically deviated toward the virtual (perceived) target position. These errors constitute the direct effect of prisms. Their correction is thought to depend on a short-latency "strategic" process that is activated by deviations from intended plans (error feedback; Redding and Wallace, 1996). In general, terminal errors are successfully corrected after a series of 10-15 trials only. Behavioral research suggests that the terminal error on a given trial may be used to correct movement on the next trial (Rossetti et al., 1993). However, further pointing movements are necessary to obtain a sustained realignment of visual and proprioceptive spatial coordinates. This is a "true" adaptation (Weiner et al., 1983) that can be demonstrated by a compensatory shift opposite to the prism deviation when subjects are asked to point straight-ahead after removal of prisms (so-called after-effects) (Redding et al., 2005).

The neural substrates underlying prism adaptation are of great importance to understand sensorimotor plasticity. Furthermore, improvement of spatial neglect after prism adaptation in stroke patients (Rossetti et al., 1998) and generalization of adaptation effects to other visuo-motor (Colent et al., 2000; Michel et

\footnotetext{
Received July 2, 2008; revised Nov. 10, 2008; accepted Nov. 14, 2008.

This work was supported by grants from Inserm and La Société Française de Médecine Physique et de Réadaptation to J.L., and Swiss National Science Foundation to P.V. (No. 3200B0-114014).

Correspondence should be addressed to Dr. Jacques Luauté, Service de Rééducation Neurologique, Mouvement et Handicap, Hospices Civils de Lyon, Hôpital Henry Gabrielle, 69230 Saint Genis Laval, France. E-mail: jacques.luaute@chu-lyon.fr.

DOI:10.1523/JNEUROSCI.3054-08.2009

Copyright $\odot 2009$ Society for Neuroscience $\quad$ 0270-6474/09/290169-10\$15.00/0
}

al., 2003; Michel et al., 2007) or non-visuomotor tasks (Striemer et al., 2006) in healthy subjects have generated a major theoretical interest because they suggest that visuomotor adaptation might modulate higher-level processes involved in spatial cognition.

A recent model (Pisella et al., 2006b) proposed that prism adaptation might critically depend on the cerebellum, in keeping with the lack of adaptation observed in patients with cerebellar lesions (Weiner et al., 1983; Pisella et al., 2005), whereas the subsequent cognitive effects might involve the posterior parietal cortex (PPC) via bottom-up signals generated by the cerebellum. An important role for PPC is also suggested by functional neuroimaging (Clower et al., 1996). In this pioneer study, PET scans were performed in healthy volunteers while prismatic deviation was reversed every five trials (left vs right), and revealed selective activation in PPC during pointing with prisms (vs without prisms), independent of the shift direction. However, because deviation was repeatedly changed, this study captured only the strategic short-term correction of visuo-motor errors before any sustained realignment of sensorimotor coordinates could be established. Another preliminary PET study on pointing with versus without prisms reported activation in the lateral cerebellum, plus ventrolateral thalamus and prefrontal cortex (Zeffiro, 1995). Sensori-motor plasticity has also been investigated using visuomotor rotation paradigms (Inoue et al., 1997; Imamizu et al., 2000; Graydon et al., 2005; Seidler et al., 2006; Girgenrath et al., $2008)$, but the large magnitude of visual rotation $\left(\geq 90^{\circ}\right)$ in these studies may involve high-level strategic control processes that are distinct from pure spatial realignment mechanisms concerned by prism displacement (Redding et al., 2005). Although one study (Diedrichsen et al., 2005) used a small rotation $\left(25^{\circ}\right.$, closer to prismatic shift), the direction was regularly reversed (clockwise 
or counterclockwise) so that the slow spatial realignment component of adaptation could not develop.

Here we examine the neural bases of sustained prism exposure with the aims to capture both the early strategic component of prism adaptation and the more progressive realignment process. We used an event-related fMRI design allowing a dynamic investigation of neural activity over the time course of prism exposure and adaptation.

\section{Materials and Methods}

Participants. We examined 14 healthy volunteers, 7 women and 7 men. Three subjects had to be excluded from the analyses because of technical problems during acquisition (two failed to correctly hear auditory instructions and behavioral responses of the third one were missing). All tested subjects (age range 19-28, mean 23) were right handed as assessed by the Edinburgh inventory (Oldfield, 1971). Eight of these subjects were right eye dominant and three left eye dominant as determined by standard methods. All participants had a normal or corrected-to-normal vision, and no history of neurological or psychiatric disorders. They all gave informed consent according to the local ethical committee regulation.

Methods. Our paradigm included all three classical phases of prism adaptation: (1) preexposure baseline; (2) active exposure to prismatic deviation over a prolonged period; (3) postexposure compensation or "deadaptation" (Redding et al., 2005). Participants had to point to visual targets with their right hand, across four consecutive fMRI runs: either without prism (runs 1 and 4), or with prism (runs 2 and 3 ) that shifted their vision $10^{\circ}$ leftward (Fig. 1). Event-related fMRI responses were obtained for each pointing trial in each condition over the whole course of adaptation (see Experimental procedures). By comparing pointing during different runs, we could distinguish the different phases of prismatic adaptation, including short-term strategic corrections and "realignment," as well as any activity related to the de-adaptation phase.

Participants were installed in the MRI scanner with a particular set-up allowing a slight anterior flexion of the head position to allow a direct vision of the target display in front of the scanner bore, with a very strict, although comfortable, fixation of head and shoulders to prevent systematic pointing-related movements (see Fig. 1b). Prismatic goggles were specifically made for this experiment using a point-topoint prismatic lens (Optiquepeter.com). The main advantage of these prisms, compared with Fresnel prisms, is the absence of any optical deformation of the environment perceived by the subject, which is thought to favor low-level sensori-motor spatial realignment (true adaptation) instead of higher-order strategic control. They were placed in front of the participant's eyes and attached to their forehead and to the headcoil. They were composed of a MRI (a)

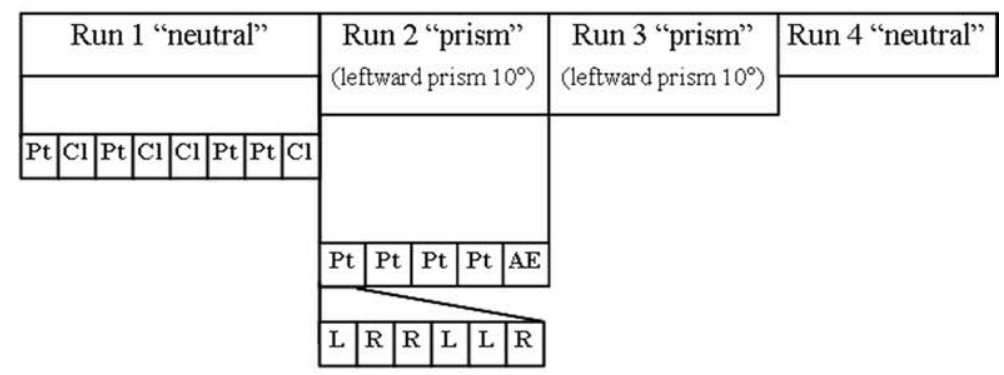

(b)

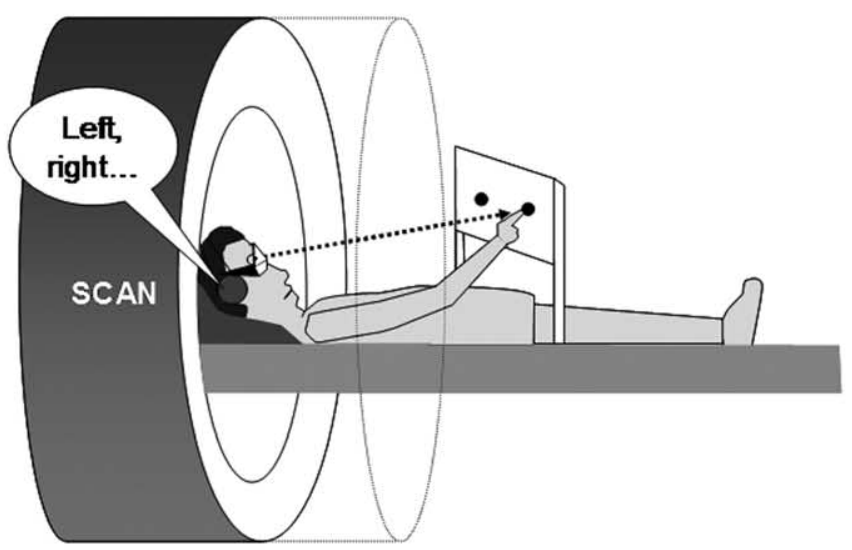

(c)

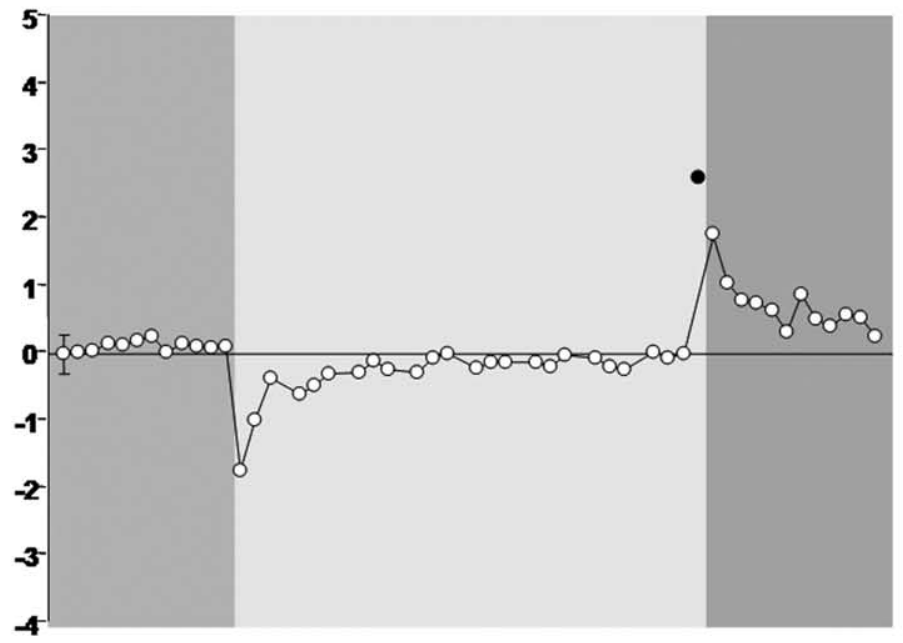

Figure 1. Experimental design and behavioral results. $\boldsymbol{a}$, Four successive fMRI runs were performed, either without prisms (run 1: before prism exposure; run 4: after prism exposure) or with prisms (run 2: early prism exposure; run 3: late prism exposure). In each run, participants pointed with the right hand to a right or left target (random order). Because the head was slightly bent forward, targets were seen in direct vision and not reflected in a mirror. In runs 1 and 4, pointing trials alternated with control blocks (clicking "Cl" on a right or left button with the right hand). At the end of run 3, a measure of prisms after-effect was performed by asking the subject to look at a target without prism and point to its remembered position with eyes closed. $A E$, After-effect; Pt, pointing; $\mathrm{Cl}$, clicking; L, left; R, right. $\boldsymbol{b}$, Set-up for the pointing task. Subjects tried to reach with the tip of their right index to one of two possible visual targets, located on the left or right side of a touch-sensitive display in the front of the scanner bore. On each trial, the target to reach was indicated by an auditory command (the word "left" or "right") through earphones. $c$, Average end-position (visual angle) of each pointing trial during fMRI (for right and left targets plotted together). Positive values refer to a right deviation, and negative values refer to a left deviation. Pointing to targets with visual feedback (empty circles) showed a classical adaptation pattern. Before prism exposure (run 1), end position was accurate (baseline). Introducing the leftward prism (run 2) induced leftward deviations (directeffect). The course of error correction comprised an early stage (during run 2) with a rapid decrease of deviations, and a later stage with return to the baseline (run 3). At the end of prism exposure, adaptation was tested by pointing without visual feedback (filled circle) to targets (left or right) seen after a transient removal of the prism. This revealed a clear rightward compensatory shift for all subjects, confirming a successful sensorimotor adaptation. After removal of the prism (run 4), a compensatory rightward shift was observed, which was then progressively corrected. 
compatible cardboard frame and two transparent glasses: one neutral (without any deviation) and one prismatic (producing a $10^{\circ}$ leftward shift of the visual wide-field). Position of the prism on the goggles was randomly assigned across participants ( 7 subjects had the prism over the right eye, 4 over the left eye). In addition, an opaque patch was put in front of the goggles' frame to allow monocular occlusion of either the right or left eye. This patch was controlled by a mechanical device such that it could be moved from one eye to the other during scanning without moving the goggles or the head. The patch was placed over the eye with the prism during runs 1 and 4 (i.e., neutral vision conditions), and over the nonprismatic neutral glass during runs 2 and 3 (i.e., prism exposure conditions).

The experiment began with two brief training sessions of pointing without prisms, outside and inside the scanner. Then participants performed 4 fMRI runs ( $\sim 7 \mathrm{~min}$ for run 1 and 4 and $\sim 6$ min for run 2 and 3 ). Runs 1 and 4 (neutral vision) consisted of 4 blocks of pointing in alternation with 4 blocks of clicking.

The pointing task required subjects to reach (with the tip of their right index) to one of two possible targets, located on the left or right side of a touch-sensitive display in front of the scanner bore. The targets were two red points horizontally aligned on either side of the display $\left(\sim 10^{\circ}\right.$ from center) and remained visible throughout the task. On each trial, the target to reach was indicated by an auditory command (the word "left" or "right") through earphones, in a random sequence ( 3 left, 3 right in each block). To ensure optimal adaptation, subjects were trained to make pointing movements as fast and accurately as possible in a single movement, with a starting hand position on their umbilicus, occluded from sight; and then to return to this position immediately after touching the target. In the clicking task, subjects were required to press (with their right index) on either the right or left button of a response-pad, fixed on their umbilicus. Instructions were identical to the pointing task, with the same auditory commands ("left" or "right") given by earphones in a random order ( 3 clicks on either side in each block).

Each task was repeated four times in each of these runs (1 and 4), resulting in 24 pointing trials ( 12 to the left target, 12 to the right) and 24 clicking trials ( 12 on the left key, 12 on the right) per run. Runs 2 and 3 (prism exposure) consisted of 4 blocks of pointing trials, alternating with short rest periods without pointing. Pointing trials were exactly identical to the other runs (see above) except for the presence of the prism. Each block included 3 right and 3 left targets, in random order. At the end of prism exposure (run 3), two adaptation test trials were performed, in which the goggles were changed to the neutral position (with the patch over the prism), and subjects were instructed to look and then point to one of the visual targets with their eyes closed. These instructions were given by earphones, without interrupting fMRI acquisition, with the following sequence: "Look at the left (or right) target; close your eyes; and now point." Participants made one pointing movement to the left target, and one to the right, in random order.

Pointing accuracy was recorded on each trial by comparing the endposition of the movement relative to the target position on the visual display. The latter consisted of a touch-sensitive surface, made with a thin soft plastic board overlaying a PC keyboard adapted for MRI compatibility. Individual keys were not visible to avoid any visual cues other than the two color targets. Pointing errors were measured in terms of the distance between the keys touched at the end of the movement and the key corresponding to the correct target position. This error was subsequently transformed in visual angle, with a deviation of 1 key $(2 \mathrm{~cm})$ corresponding to $\sim 2^{\circ}$ (given a distance between the subject's eyes and visual display of $\sim 55 \mathrm{~cm}$ ). A more precise spatial discrimination of errors was obtained by recording all keys simultaneously pressed by the subject, and by calculating the final end-position as the average key coordinates (which yielded a resolution of $0.5^{\circ}$ in the horizontal axis given the lateral shift of keys between rows). The size of terminal pointing errors allowed us to derive different behavioral indices for each pointing trial, used as independent predictor in our parametric analyses of fMRI data (see below). The error size index was determined as the absolute magnitude of terminal error on a given trial (i.e., with negative to positive values for leftward to rightward deviations, respectively). The error correction index was determined as the amplitude of pointing adjustment occurring between the preceding trial and the current trial (error correction on trial $n=$ amplitude of the terminal error on trial $n-1$ minus the terminal error on trial $n$ ). Error correction was set to zero for the first trial of each pointing block given that no correction could occur in this case.

MRI scanning. MRI data were acquired in a $1.5-\mathrm{T}$ whole-body INTERA system (Philips Medical Systems), using the standard head-coil configuration. For each participant, structural images were acquired with a 3-D-GRE T1-weighted sequence $(\mathrm{FOV}=250 \mathrm{~mm}$, TR/TE/Flip $=15$ $\mathrm{ms} / 5.0 \mathrm{~ms} / 30^{\circ}$; matrix $=256 \times 256$, slice thickness $\left.=1.25 \mathrm{~mm}\right)$ and functional images with a GRE EPI sequence (TR/TE/Flip $=2500 \mathrm{~ms} / 40$ $\mathrm{ms} / 80^{\circ}, \mathrm{FOV}=250 \mathrm{~mm}$, matrix $\left.=128 \times 128\right)$. Each functional image comprised 30 contiguous $4.5 \mathrm{~mm}$ axial slices $(2 \times 2 \mathrm{~mm}$ in plane), oriented parallel to the inferior edge of the occipital and temporal lobes, and covering the whole brain from cerebellum to parietal cortex. For the main experiment, a total of 636 functional images were acquired in four scanning runs, each separated by a very brief pause, with an effective repetition time of $2.5 \mathrm{~s}$.

Data analysis. Images were processed and analyzed using SPM2 (Wellcome Department of Imaging Neuroscience). All functional volumes were realigned to the first volume (Friston et al., 1995a), spatially normalized to a standard template with a resampled voxel size of $2 \times 2 \times 2$ $\mathrm{mm}$, and smoothed using a Gaussian Kernel with a full width at half maximum (FWHM) of $8 \mathrm{~mm}$. To remove low-frequency drifts from the data, high-pass temporal filtering with a cutoff of $128 \mathrm{~s}$ was applied. Statistical analysis was performed using the general linear model (Friston et al., 1995b) for event-related designs as implemented in SPM2. In our main analysis, each pointing and clicking trial was modeled by convolving a $\delta$ function at the trial onset (i.e., auditory command) with a canonical hemodynamic response function (HRF). Error size and error correction measured on each pointing trial were included in the same design matrix as additional parametric regressors, also convolved with the HRF. Because these two regressors were effectively orthogonalized in SPM analysis, we could obtain a measure of their independent contribution to the neural responses on a trial-by-trial basis in each subject. The orthogonality between error and correction parameters was confirmed by computing the absolute cosine values of their angle for each participant: mean $0.0676(\mathrm{SD}=0.0244)$ and $0.0778(\mathrm{SD}=0.0406)$ for runs 2 and 3 , respectively (values close to zero indicate orthogonality, whereas values close to 1 indicate colinearity), corresponding to nonsignificant correlation coefficients between these two parametric regressors $(r<1, p>0.27$ for both runs). In addition, residual effects of head motion were corrected by including the six estimated motion parameters for each subject as regressors of no interest in the design matrix.

The general linear model was then used to generate parameter estimates of activity at each voxel, for each regressor and each participant. Contrasts images were calculated by applying appropriate linear contrasts to the parameters estimates for the regressors of each event. These contrast images were then entered into one-sample $t$ tests across the 11 participants separately to perform random-effects group analyses. All statistical analyses were performed on a voxelwise basis across the whole brain. We report results at $p<0.001$ uncorrected for multiple comparisons and cluster size $>10$. Location of activations in the cerebral cortex was determined on the average brain template of the 11 subjects, according to the stereotaxic coordinate system of the standard MNI template; location of activations in the brainstem and cerebellum was determined according to Schmahmann's atlas (Schmahmann et al., 1999).

In a complementary SPM analysis, to estimate the time course of activity on a trial-by-trial basis, we modeled individual pointing trials during prism exposure (runs 2 and 3 ) as separate events in a new designmatrix, including again motion parameters of no interest, and then applied the general linear model to generate new parameter estimates of activity at each voxel, for each regressor and each participant. These parameters were pooled for left and right targets and averaged for pairs of successive trials to smooth the time course curves. Beta values (i.e., parameter estimates of neural activity corresponding to each regressor in the general linear model) obtained with this analysis were extracted and averaged from clusters of three specific regions-of-interest (ROIs) that were previously identified in our main SPM analysis (antIPS, POS, and cerebellum). 


\begin{tabular}{|c|c|c|c|c|c|c|c|c|}
\hline \multirow[b]{2}{*}{ Area } & \multirow[b]{2}{*}{ Side } & \multirow[b]{2}{*}{$B A$} & \multirow[b]{2}{*}{ Cluster size } & \multirow[b]{2}{*}{$T$} & \multirow[b]{2}{*}{ Z } & \multicolumn{3}{|c|}{ Coordinates of peak activation (mm) } \\
\hline & & & & & & $x$ & $y$ & $z$ \\
\hline Cerebellum vermis & & Vermis & 416 & 11.35 & 5.03 & 2 & -78 & -26 \\
\hline Cerebellum hemisphere & $\mathrm{R}$ & Lobule 4/5 & 470 & 8.85 & 4.57 & 20 & -32 & -28 \\
\hline Cerebellum hemisphere & $\mathrm{R}$ & Lobule 8R & 50 & 7.93 & 4.36 & 26 & -40 & -50 \\
\hline Cerebellum hemisphere & $\mathrm{L}$ & Lobule 4/5 & 106 & 9.23 & 4.65 & -24 & -24 & -32 \\
\hline Cerebellum hemisphere & $\mathrm{L}$ & Crus II & 46 & 5.8 & 3.75 & -32 & -80 & -50 \\
\hline Cerebellum hemisphere & $\mathrm{L}$ & Lobule 8L & 28 & 4.63 & 3.31 & -32 & -46 & -44 \\
\hline Motor cortex & $\mathrm{L}$ & 4 & 810 & 9.33 & 4.67 & -12 & -48 & 62 \\
\hline Motor cortex & $\mathrm{R}$ & 4 & 16 & 5.52 & 3.66 & 30 & -30 & 60 \\
\hline Premotor cortex & $\mathrm{R}$ & 6 & 28 & 5.14 & 3.52 & 26 & -20 & 68 \\
\hline SMA & $\mathrm{R}$ & 6 & 13 & 4.85 & 3.4 & 8 & -16 & 58 \\
\hline SMA & $\mathrm{L}$ & 6 & 80 & 5.51 & 3.65 & -8 & -26 & 50 \\
\hline Somato-sensory cortex & $\mathrm{L}$ & 3 & 30 & 5.37 & 3.6 & -36 & -20 & 48 \\
\hline Occipital & $\mathrm{R}$ & $18 / 19$ & 73 & 5.56 & 3.67 & 6 & -86 & 6 \\
\hline Occipital & $\mathrm{L}$ & $18 / 19$ & 15 & 4.67 & 3.33 & -32 & -90 & 16 \\
\hline Occipito-temporal & $\mathrm{R}$ & 37 & 253 & 8.69 & 4.54 & 52 & -68 & 4 \\
\hline Occipito-temporal & $\mathrm{L}$ & 19 & 65 & 6.86 & 4.09 & -44 & -72 & -14 \\
\hline 0ccipito-parietal & $\mathrm{R}$ & & 218 & 5.89 & 3.78 & 26 & -78 & 40 \\
\hline 0ccipito-parietal & $\mathrm{L}$ & 19 & 181 & 5.49 & 3.65 & -10 & -82 & 44 \\
\hline SPL & $\mathrm{R}$ & $5 / 7$ & 60 & 5.58 & 3.68 & 18 & -62 & 64 \\
\hline $\mathrm{IPL}$ & $\mathrm{R}$ & 39 & 14 & 4.56 & 3.28 & 40 & -76 & 18 \\
\hline Parahippocampal gyrus & $\mathrm{L}$ & 36 & 53 & 8.26 & 4.44 & -22 & 0 & -36 \\
\hline
\end{tabular}

All $p$ values $<0.001$ (uncorrected), cluster size $>10$ voxels. IPL, Inferior parietal lobule; SPL, superior parietal lobule; SMA, supplementary motor area; L, left; $R$, right; BA, Brodmann area.

\section{Results}

\section{Behavioral performance}

Pointing accuracy was determined by the distance between the target and terminal location on the touch-pad. We compared the different pointing conditions by ANOVA on the mean terminal deviation using adaptation phase (runs 1-4) as factor. As can be seen in Figure $1 c$, the main effect of phase was highly significant $\left[F_{(3,33)}=39.04 ; p<0.0001\right]$, with Scheffé post hoc tests showing a difference between run 2 and run 4 relative to run 1 (both $p<$ $0.0002)$. There was no significant difference between run 3 and run 1, confirming that terminal position returned to baseline values (correct target location) during run 3.

To assess the time course of error correction, we also performed trial-by-trial one-sample $t$ tests comparing the average end-position of each pointing (left and right trials together) with the real position of the target (reaching the latter corresponding to zero deviation). This showed no significant deviation during run 1. During run 2, the maximum pointing deviation was observed on the first trial (mean deviation $=1.7^{\circ}$ to the left from the real target position). The end-position of the first 12 trials plus the leighth trial were significantly different from the real target position, whereas the last 6 trials of run 2 and all subsequent trials of run 3 were not different from the real target position. In run 4, the end-position was again significantly different from correct position for all trials except the last two, demonstrating a sustained compensatory effect after prism exposure.

In addition, a direct measure of adaptation strength was obtained at the end of prism exposure by test trials performed without prism, but eyes closed (see Materials and Methods) (Fig. 1c). These test trials confirmed a reliable sustained adaptation, with highly significant rightward shift (mean deviation $=2.5^{\circ}$ opposite to the optical deviation) between end-position and real target position (i.e., clear after-effects).

\section{Brain imaging results}

Control of visuo-motor actions: pointing $>$ clicking

We first identified brain regions implicated in the visual guidance of motor actions by contrasting all pointing trials made without prisms, relative to all clicking trials performed in the same runs (pointing run $1+$ pointing run $4>$ clicking run $1+$ clicking run 4 ). Thus, any general cognitive processes activated by instructions, target selection, and/or motor decisions were canceled out in this contrast. This analysis identified a network of regions implicated in motor and proprioceptive control, including the contralateral primary motor cortex, overlapping with an area associated with elbow movements (Alkadhi et al., 2002); cerebellum (vermis and both hemispheres); bilateral supplementary motor area (SMA); and contralateral somatosensory cortex (Table 1). Activations were also observed in superior parietal regions implicated in visuo-motor action (Buneo and Andersen, 2006) including the posterior parieto-occipital sulcus (POS) and intraparietal sulcus (IPS). These regions were used to define a functional mask (at $p<0.05$ ) of regions of interest (ROIs), specifically associated with visuo-motor control, which we could then test for changes during prism adaptation in subsequent analyses (see below).

Sustained exposure to prism deviation: prism $>$ neutral

Brain regions recruited during the whole period of pointing under exposure to prismatic displacement were identified by contrasting all pointing trials in runs with prisms, relative to the first run without prism (i.e., [pointing runs $2+3$ ] $>$ [pointing run 1] $\times 2)$. This revealed selective activation in both the right and left superior temporal gyrus (STG) extending to the posteriorsuperior temporal sulci (STS) (Table 2, Fig. 2). When using an inclusive mask of ROIs previously defined as specific to visuomotor actions (i.e., pointing $>$ clicking, see above), no significant overlap was found with the activated loci in STG/STS. This result argues against a role of these areas for the direct visuo-motor command of pointing, but rather suggests a contribution to higher nonmotor function that can be modified by prism adaptation; see review in Michel (2006). Moreover, when comparing activation in these temporal areas across the two successive prism runs, both the left and right STG/STS showed a further increase for pointing during run 3 compared with run 2 (Fig. 2) ( $T>4.93$, $p<0.001$ for both sides). This result further supports the hypoth- 
Table 2. Brain regions activated during prism adaptation

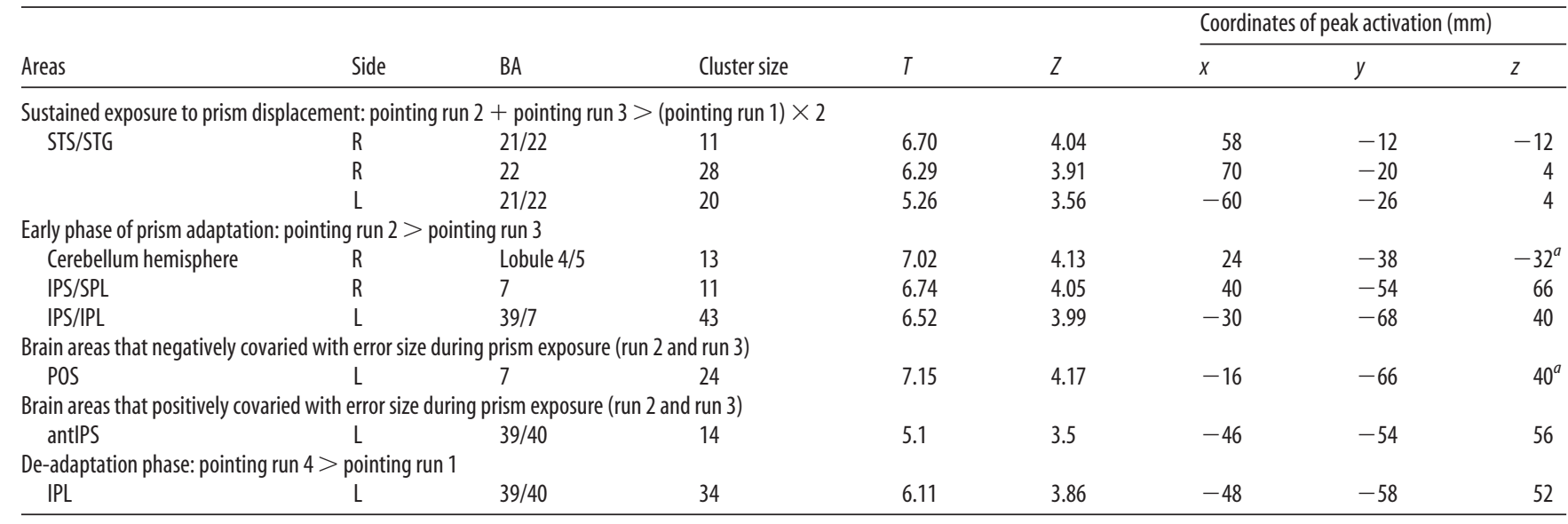

All $p$ values $<0.001$ (uncorrected), cluster size $>10$ voxels. STS, Superior temporal sulcus; STG, superior temporal gyrus; PPC, posterior parietal cortex; IPS, intraparietal sulcus; antIPS, anterior bank of the intra-parietal sulcus; IPL, inferio parietal lobule; SPL, superior parietal lobule.

${ }^{a}$ Regions overlapping with visuo-motor activations in the pointing $>$ clicking contrast (functional mask at $p<0.05$, voxel $>10$ ).

(a)

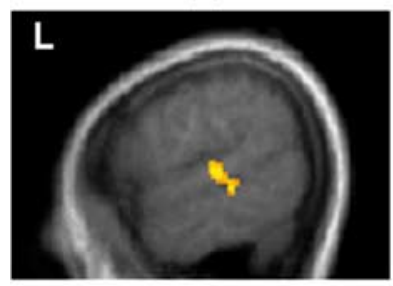

(b)

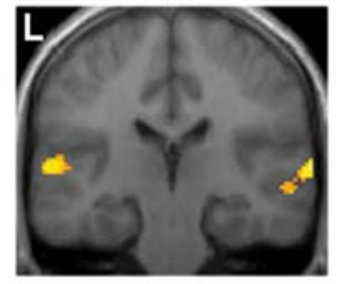

(d)
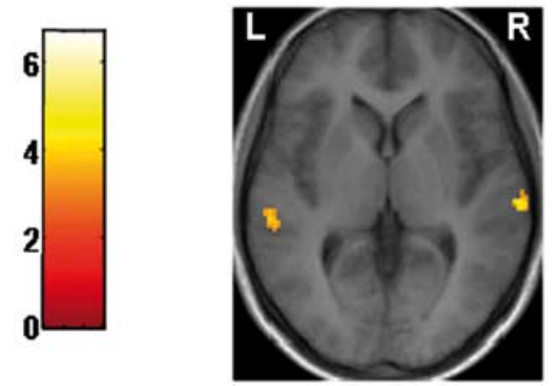

(c)

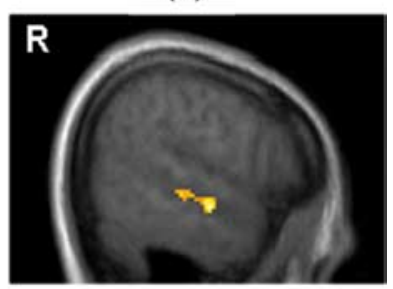

(e)

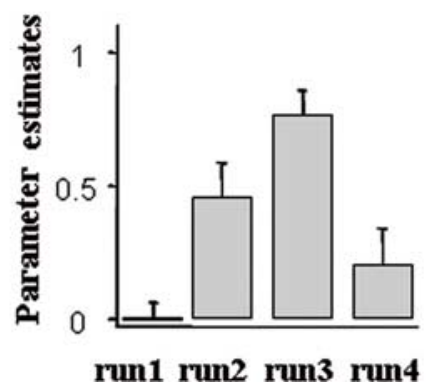

Figure 2. Brain areas activated by sustained exposure to prism deviation. $\boldsymbol{a}-\boldsymbol{c}$, Activation in the left and right superior temporal cortex revealed by contrasting pointing during run 2 and run 3 (with prism) relative to pointing during run 1 (baseline, without prism), displayed on the average brain of the 11 subjects: $\boldsymbol{a}$, left sagittal section at $x=-60 ; \boldsymbol{b}$, right sagittal section at $x=58 ; \boldsymbol{c}$, coronal section at $y=-22 ; \boldsymbol{d}$, horizontal section at $z=0 . \boldsymbol{e}$, Parameter estimates of activity (betas, mean \pm SE) in the right STS/STG $(x, y, z=58,-12,-12)$ for pointing trials across the four successive runs ( \pm SE). A similar pattern was found in left STS/STG.

esis that activation within the STG/STS is not directly associated with sensorimotor plasticity but rather reflects higher-order processes induced by prism adaptation.

Exposure to prism deviation: early versus late phase

To determine brain areas more specifically implicated during the early stage of prism exposure, we contrasted pointing trials performed during the second versus the third fMRI runs (pointing in run $2>$ pointing in run 3 , all executed in presence of the exact same prismatic displacement) (Fig. 1). This comparison identified a network of areas including the right cerebellum hemisphere (lobule 4 and 5), the right superior parietal lobule (SPL) extending to the intraparietal sulcus (IPS), and the left posterior parietal cortex extending to IPS and inferior parietal lobule (IPL) (Table
2, Fig. 3). Among these regions, only activation in the right cerebellum overlapped with the ROI mask previously defined as specific to visuomotor actions (pointing > clicking), suggesting a role in both the visuo-motor control of pointing and the early exposure to prism deviation. Unlike STG/STS, none of these parietal and cerebellar regions showed any progressive increases in activation during pointing in run 3 relative to run 2, but a significant reduction instead.

We corroborated these data by performing an additional parametric analysis based on the magnitude of terminal pointing errors, which progressively decreased over successive trials during prism exposure (runs 2 and 3). This analysis tested for any voxels across the whole brain in which eventrelated activity during pointing showed a linear parametric modulation as a function of deviation magnitude, therefore allowing us to identify regions in which activity covaried either positively or negatively with error magnitude on each individual trial (see Materials and Methods) (Table 2, Fig. 4a). A significant positive correlation (i.e., voxels with activity increasing in proportion to the magnitude of deviation) across all successive trials under prism (runs 2 and 3 ) was found in the left anterior intraparietal sulcus (antIPS). Another activation was observed in the anterior cingulate cortex (ACC) $(x, y, z=-2$, $30,22, p<0.001$, but with a cluster size below our threshold of 10 voxels). However, a significant negative correlation (i.e., voxels with activity decreasing in proportion to the magnitude of deviation) across all successive trials in runs 2 and 3 was found in the left parieto-occipital sulcus (POS). This cluster was located on the supero-anterior bank of the sulcus. When comparing these regions with the ROI mask previously defined by specific visuo-motor activity (pointing > clicking), only the left POS (negative covariation) showed a significant overlap, suggesting a direct role in visuo-motor control.

Because the correlation between parietal activation and error 
size during adaptation might reflect neural processes engaged by either detection or correction of errors (both being greater during early than late stages of prism exposure), our parametric analysis also included an index of error correction, defined as the difference in error size on trial $n$ relative to the preceding trial $n-1$ (see Materials and Methods), in addition to the absolute error size index used above. For both antIPS and POS clusters identified above, we compared the values of these two different parametric regressors, estimating the modulation of activity during pointing as a function of either error magnitude or correction magnitude on the current trial (as determined by separate parametric factors in a single SPM analysis). This comparison revealed that activity in left POS was primarily modulated by error correction on the current trial, more than by error size; whereas conversely, activity in left antIPS was modulated by error size more than by correction (Fig. $4 b$ ). In other words, a modulation of POS by behavioral performance was better accounted for by error correction than by error size on a given trial; and conversely a modulation of antIPS by performance was better accounted for by error size than by error correction. ANOVA on the parametric estimates obtained for each modulation type (error size and correction) and each region confirmed a highly significant interaction between modulation type and region $\left[F_{(1,20)}=21.35 ; p<0.001\right]$. These data provide further evidence that the positive covariation of antIPS activity with pointing errors during prism exposure (Fig. $4 a$ ) is related to the detection of deviation, whereas the negative covariation of POS with pointing errors is directly related to the correction of deviation.

\section{Trial-by-trial analysis of adaptation}

Given the dynamic nature of adaptation, we performed a separate analysis to calculate parameter estimates of cerebral activity on a trial-by-trial basis, and thus determine more precisely the time course of changes in specific regions of interest (ROIs). In this complementary analysis, individual pointing trials during prism exposure in runs 2 and 3 (averaged across pairs of left and right targets) were modeled by separate regressors in SPM (see Materials and Methods). Parameter estimates were then extracted for these successive trials from ROIs in the left antIPS and left POS (which were defined by our previous parametric analysis on error size), as well as in the right cerebellum (which was activated in the first run of prism exposure, see above). The time course of activation in these three regions is plotted in Figure 5, and shows differential activation across the successive trials performed during the first run of prism exposure (while error and correction of the error took place). During the first 12 trials, antIPS progressively decreased its activity and POS progressively increased activity, before both regions reached a relative plateau. Whereas activity in POS then remained relatively stable between trials 12 (d) IPL

(b) IPS/SPL

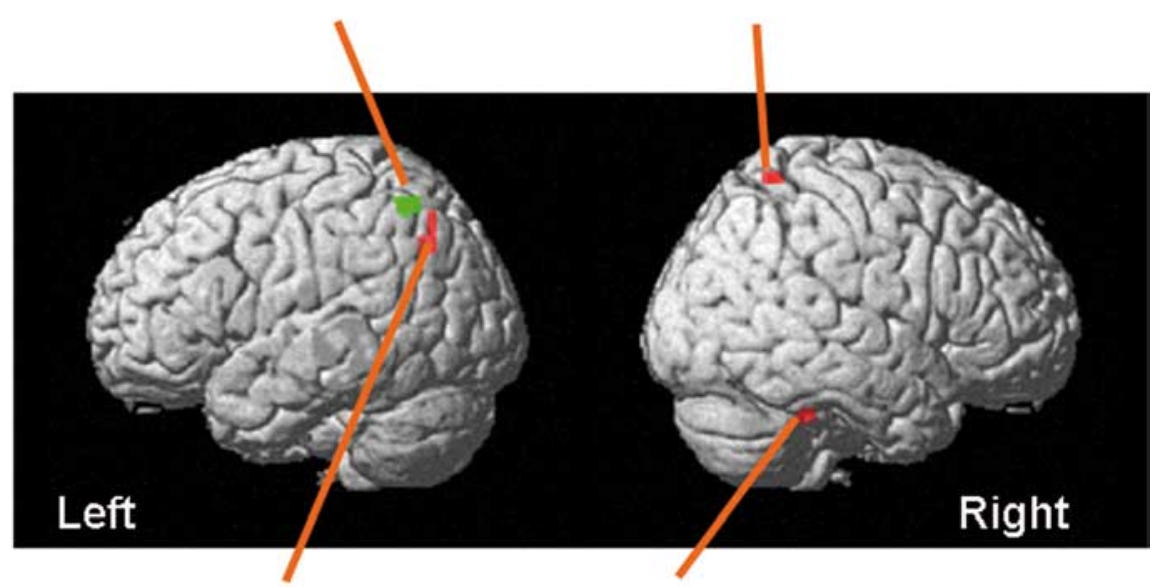

(e) IPL
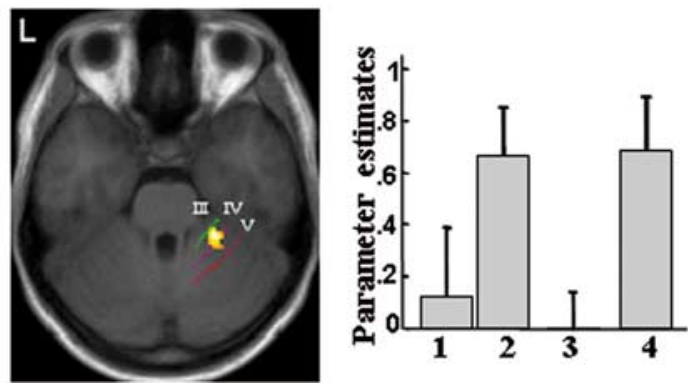

(c) cerebellum

Figure 3. $\quad \boldsymbol{a}-\boldsymbol{d}$, Brain areas activated by early adaptation to prisms and postprism de-adaptation. $\boldsymbol{a}, \boldsymbol{b}$, Activations in right and left parietal cortex revealed by contrasting pointing in the early versus late phase of adaptation (run $2>$ run 3 ), displayed in red

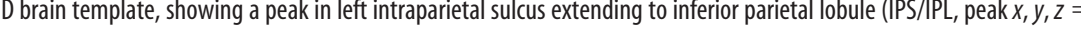
c, Activation in right cerebellum [peak $x, y, z=24,-38,-32$, corresponding to lobule IV and V according to Schmahmann at $z=46(\boldsymbol{a})$ and at $z=-32$ (c). d, Activation in the left parietal cortex was also found during the de-adaptation phase by contrasting pointing trials during run 4 (without prism but postprism exposure) relative to pointing during run 1 (baseline without prism), displayed in green (peak $x, y, z=-48,-58,52$ ).

18, antIPS showed further fluctuations until trials 18-20 before declining more completely. The right cerebellum showed a more progressive increase over trials, reaching a later peak around trial 16 , and then gradually decreased during the last 6 trials. The time course of these activations were parallel to the pattern of deviation and correction in pointing performance over the same trials, because the greatest improvement in accuracy took place from trial 1-12 and returned to a steady baseline performance after trial 18 (Fig. 5) (see Behavioral results).

\section{De-adaptation}

Finally, we tested for brain regions involved in the de-adaptation process (after-effects), after removal of the prism after successful adaptation (Table 2). By contrasting pointing trials without prism in the last run relative to the first run (run $4>$ run 1), we found selective increases in the left inferior parietal lobule extending to the antIPS, close to areas activated during early versus late phase of prism exposure (Fig. $3 d$ ) as well as those showing a positive correlation with pointing errors in parametric analysis above (Fig. 4a). This is consistent with the marked deviation errors observed behaviorally during initial pointing trials in the de-adaptation run (Fig. 3e). 
(a)

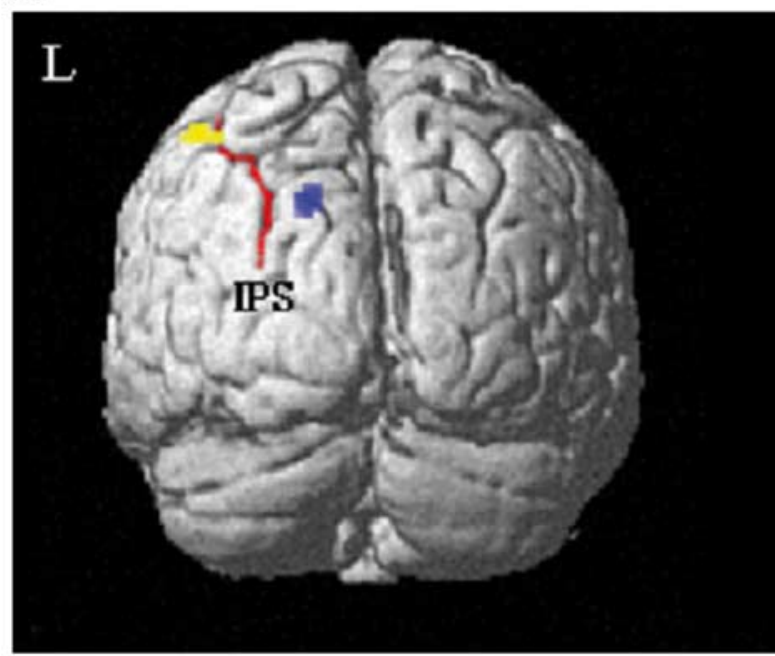

(b)
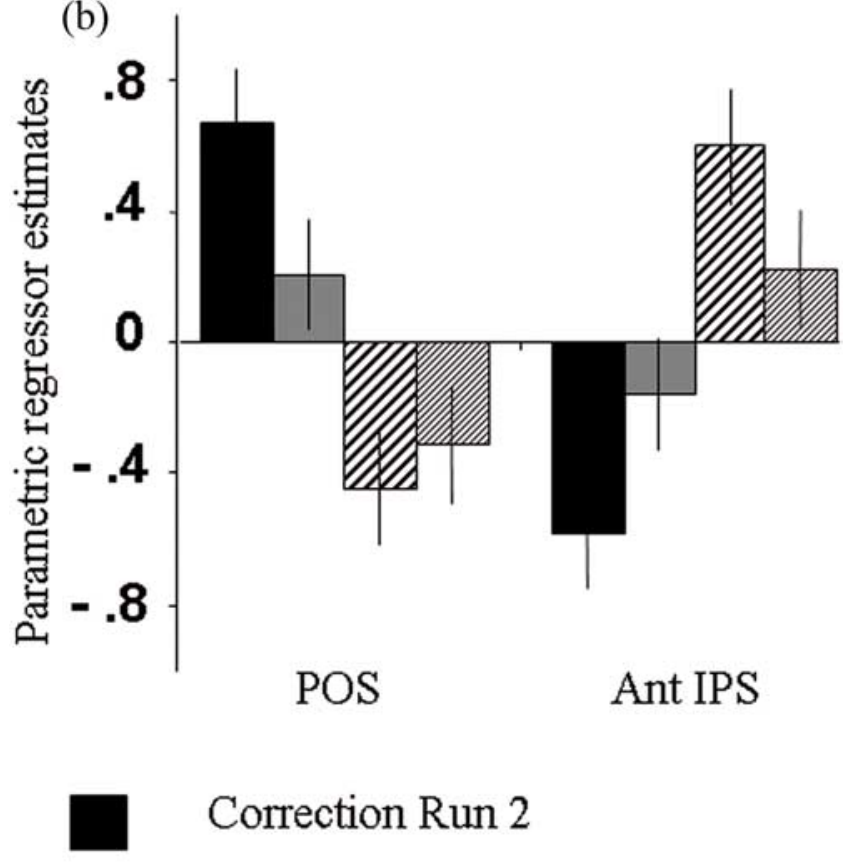

\section{Correction Run 2}

\section{Correction Run 3}

\section{Error Run 2}

\section{Error Run 3}

Figure 4. Parametric modulation of activation in relation to pointing error size during prism exposure. $\boldsymbol{a}$, Areas showing increases proportional to error size were found in the left anterior IPS (antIPS, displayed in yellow on a 3D brain template, peak $x, y, z=-46,-54,56$ ). Conversely, areas showing activity inversely proportional to error size were found in the left parietooccipital sulcus (POS, displayed in blue, peak $x, y, z=-16,-66,40)$. $\boldsymbol{b}$, Mean estimates of parametric modulation for these two clusters (betas for parametric factors as computed by SPM regression analysis), demonstrating a greater effect of error size for activity in antIPS, but a greater effect of correction size (difference in error size between the preceding and current trials) for activity in POS. Both parameter were also greater during run 2 than run 3.

\section{Discussion}

Our study reveals for the first time the neural substrates of prism adaptation in healthy subjects, by tracking dynamic changes in behavior and brain activity over the course of both sustained prism exposure and de-adaptation. Several distinct areas were activated, reflecting specific processes involved in prism adaptation, from initial pointing errors to successful spatial realignment, as well as possible influences on spatial cognition.

We should note that our paradigm implied an alternation of monocular vision across pointing conditions (one eye with prisms, runs 1 and 4; vs the other eye without prisms, runs 2 and 3 ). However, only one contrast (Prism $>$ Neutral) was potentially affected by this bias, and the bilateral and symmetrical activation (in superior temporal cortex) revealed by this comparison is unlikely to be explained by monocular alternation.

\section{Posterior parietal cortex}

In posterior parietal cortex (PPC), the left parieto-occipital sulcus (POS) and left anterior intraparietal sulcus (antIPS) showed a distinct profile of activation suggesting separate roles during prism exposure. We found that activity in POS was related to both reaching and correction magnitude on the current trial. Thus, POS activity was low in the first prism trials (with large terminal errors), and then progressively increased until errors became reduced ( $\sim 10$ trials). This region was already reported during reaching (Connolly et al., 2003; Medendorp et al., 2003) or visuo-motor tracking (Diedrichsen et al., 2005). Moreover, parieto-occipital lesions typically produce optic ataxia, an inability to point to visual targets in peripheral vision (Karnath and Perenin, 2005; Pisella et al., 2006a). Together, these data indicate that POS might be responsible for adjusting movement plans based on new visual inputs after displacement by prism, and thus underlie successful correction during the early phase of prism exposure. A neighboring region was also selectively activated in a delayed mental rotation task (Suchan et al., 2002). This result supports the hypothesis that POS may compute the correction for the next trial through a deliberate mental translation (Rossetti et al., 1993), and hence contribute to the strategic component of prism adaptation.

In contrast, the left antIPS was activated in direct proportion to pointing deviation, as demonstrated by a positive parametric correlation between the trial-by-trial error size and activity in this region; whereas there was no correlation with the magnitude of correction on the current trial (unlike POS). These data suggest a crucial role for this region in the error detection process, consistent with a previous PET study reporting similar activation during a reaching task with jumping targets (Desmurget et al., 2001). Our results are also consistent with PET results showing a selective activation of IPL during pointing while prisms were reversed (left to right) every five trials (Clower et al., 1996). However, the latter design was likely to induce a state of continuous error production (similar to the early prism exposure and final deadaptation phases of our study). Our new data suggest that this activation may not underlie true adaptation per se (i.e., realignment of visual-proprioceptive coordinates), but only the error detection process necessary for the initial strategic corrections. This interpretation is also compatible with a case study showing impairment in error correction during prism exposure, but preserved adaptation, after bilateral parietal lesion (Pisella et al., 2004).

Finally, an activation in right intraparietal cortex was also observed during the early exposure phase, when comparing the first versus second runs of pointing with prisms. Given the major role of right $\mathrm{PPC}$ in visuo-spatial processing and attention (Mesulam, 1999), this finding may further argue for a visuo-spatial component in the initial error signal generated by prism exposure. 


\section{(a) Behavioral response}

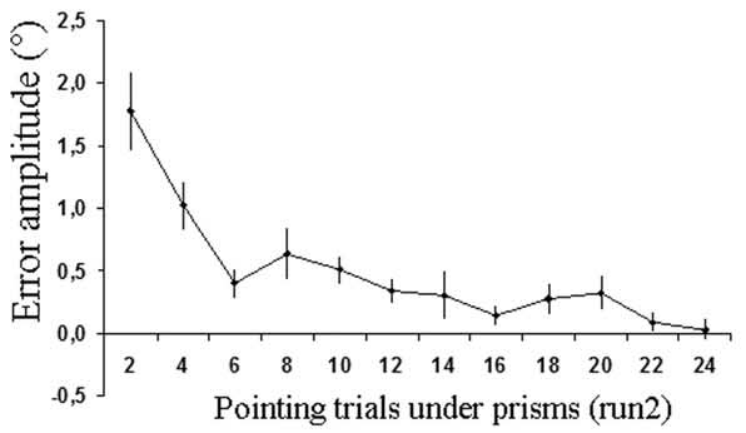

(b) Ant IPS (xyz $=-48-5852)$

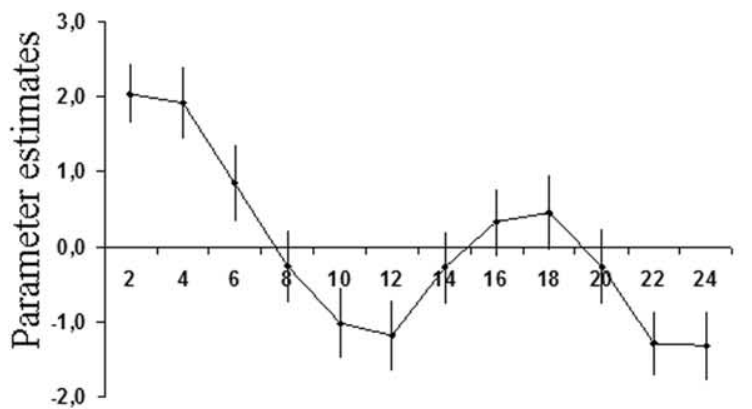

(c) $\operatorname{POS}(\mathrm{xyz}=-16-6640)$

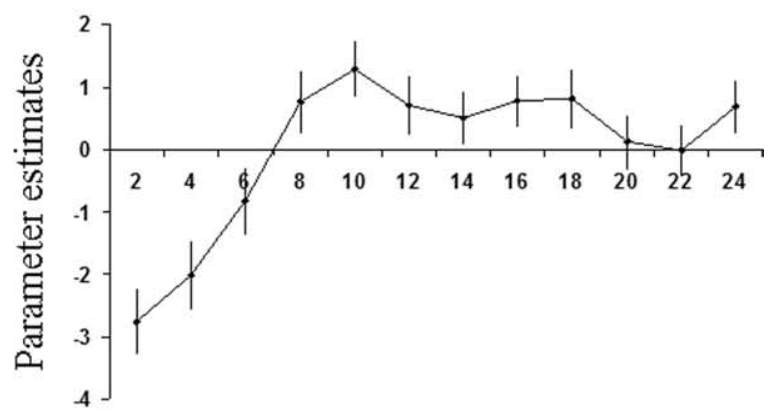

(d) Cerebellum (xyz $=24-38-32)$

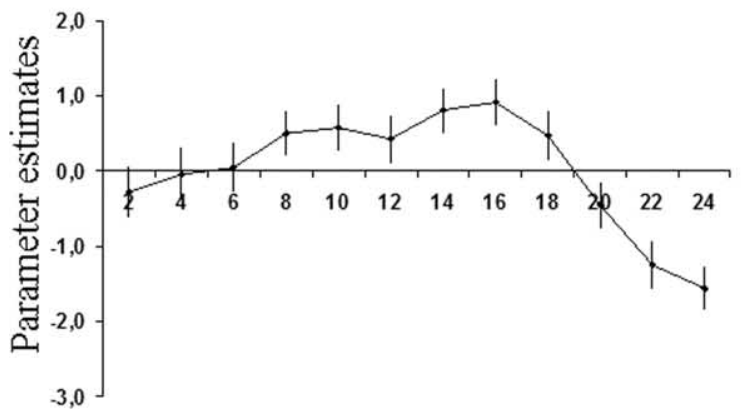

Figure 5. Time course of changes in behavioral and neural effects during early prism exposure. $\boldsymbol{a}$, Average pointing errors (in degrees of visual angle, mean $\pm \mathrm{SE}$ ) for successive trials after prism exposure (run 2). Data are pooled for left and right targets and averaged for pairs of consecutive trials. A first phase with rapid correction in the first 12 trials is followed by slower improvement, until returning to baseline performance in the last 6 trials. $\boldsymbol{b}$ - $\boldsymbol{d}$, Parameter estimates of activity for the same trials as calculated by a complementary event-related analysis in SPM using a trial-by-trial model (mean betas \pm SE), extracted for left antIPS (b), left POS (c), and right cerebellum (d). AntIPS showed rapid decrease of activity in the first 12 trials, followed by some fluctuations until reaching minimal activity in the last 6 trials. POS activity showed rapid increases in the first 9-10 trials and then reached a plateau. Right cerebellum showed more progressive increases until trial 16 and then rapidly decreased to reach minimal activity in the last 6 trials.

\section{Cerebellum}

Activation of lobules IV and V in the right cerebellum was also greater in the first run of prism exposure, relative to the second run, and overlapped with visuo-motor activity associated with pointing (relative to clicking). This is highly consistent with an implication of this region in visually directed movements (Stein, 1986; Bursztyn et al., 2006) and eye-hand coordination (Miall et al., 2000). A similar activation was found in the late stage of a visual rotation tracking task (Imamizu et al., 2000), and attributed to the acquisition of a new internal model after visual perturbation. The cerebellum involvement in prism adaptation is further supported by lesion-studies in both monkey (Baizer et al., 1999) and man (Weiner et al., 1983; Martin et al., 1996; Pisella et al., 2005). Interestingly, our trial-by-trial analysis revealed that cerebellum activity increased progressively during the early phase of prism exposure, and remained high for a longer period than the initial rapid improvement in pointing accuracy.

Although our trial-by-trial analysis is essentially descriptive and must be taken with caution because of inherent variability of fMRI signal, this dynamic profile in key regions of the cerebellum (and parietal cortex) shows a remarkable convergence between behavioral and neural data.

We suggest that the cerebellum is particularly involved in the realignment process that is necessary to re-establish a correct spatial mapping among visuo-motor and sensorimotor coordinates systems, so as to adapt to the optical shift. This process, which may result in the acquisition of a new internal model, is slower than the initial (more strategic) error correction and requires more pointing movements to become established (Redding et al., 2005). The relatively prolonged increases in cerebellar activity during prism exposure (until a stable correction of pointing errors) seem consistent with a critical function providing bottom-up signals to cortical areas responsible for the cognitive effects of prism adaptation.

\section{Superior temporal cortex}

Another major finding of our study was the selective and bilateral activation of the superior temporal sulcus (STS), extending into the superior temporal gyrus, associated with sustained prism exposure. STS increases arose only during the later stage, when pointing deviation was fully compensated and errors completely abolished. Such activity was further enhanced in the second relative to the first run with prisms, but unrelated to trial-by-trial adjustment of performance.

This result suggests an important role for STS/STG in the slow realignment process and longer-lasting changes induced by prisms. Whereas a previous study of medial-temporal lobectomy patients showed preserved prism adaptation (Canavan et al., 1990), our results provide the first direct evidence for a major role of lateral temporal regions in long-term effects of prism adaptation that might underlie changes in spatial cognition. Studies in monkeys have shown that STS and posterior-superior temporal regions receive multimodal inputs and make important contributions to cross-modal integration (Bruce et al., 1981). Thus, STS/STG is ideally suited to link multiple sensorimotor signals in a common spatial register.

These data provide new support for an implication of the superior temporal lobe in spatial cognition (Karnath et al., 2001), an issue that has been intensely debated in recent years (Halligan et al., 2003). Lesion data suggest that right temporal damage may be significantly more frequent in patients with left spatial neglect than in patients without neglect (Karnath et al., 2004), with a maximal lesion overlap being remarkably close to the right STS 
activation found in our study $(x, y, z=64-248$ vs 70-20 4, respectively). We therefore conclude that the progressive increase of STS activity during prism exposure might reflect changes in a cross-modal or supramodal system that could subserve the effects of prism adaptation on spatial cognition, although other target areas may potentially also contribute (Striemer et al., 2008).

\section{Implications for theories of visuomotor adaptation and spatial cognition}

By identifying the neural networks underlying different components of prism adaptation and their dynamical time course, our results provide new insights on the mechanisms by which visuomotor plasticity may interact with spatial cognition. Based on our results, we propose that the initial short-term strategic processes implicate error detection signals in anterior IPS and error correction plans in POS; whereas the slower realignment processes implicate the cerebellum at which different spatial maps for eye and hand sensorimotor coordinates can be integrated and realigned. In contrast, the more delayed activity observed within STS/STG could mediate the cognitive effects of prism adaptation. The latter activation might be modulated by sustained activity in the cerebellum. Hence, our results support the hypothesis that prism adaptation can modulate cortical areas implicated in spatial cognition through a bottom-up signal generated in the cerebellum (Rode et al., 2003; Pisella et al., 2006b), albeit a key role of temporal cortex was previously not suspected. Anatomical connections between the temporal cortex and the cerebellum remain poorly known but might participate to cognitive functions associated with cerebellum (Ramnani, 2006).

How sensorimotor plasticity induced by prism exposure can improve spatial neglect remains largely unknown. Our results add new support to the hypothesis that such therapeutic effects may operate by recruiting or inhibiting intact cortical areas (Pisella et al., 2006b). Among these areas, the temporal cortex of the intact left hemisphere may be responsible for improving spatial output subsequent to prism adaptation. This hypothesis is consistent with a recent PET study in neglect patients (Luauté et al., 2006), in which changes in left temporal cortex were correlated with neglect improvement after prism adaptation. Furthermore, delayed activation in temporal areas, as observed here, may contribute to consolidate these new spatial representations and explain why the improvement of spatial neglect increased after a delay of $2 \mathrm{~h}$ after prism exposure (Rossetti et al., 1998).

\section{References}

Alkadhi H, Crelier GR, Boendermaker SH, Golay X, Hepp-Reymond MC, Kollias SS (2002) Reproducibility of primary motor cortex somatotopy under controlled conditions. Am J Neuroradiol 23:1524-1532.

Baizer JS, Kralj-Hans I, Glickstein M (1999) Cerebellar lesions and prism adaptation in macaque monkeys. J Neurophysiol 81:1960-1965.

Bruce C, Desimone R, Gross CG (1981) Visual properties of neurons in a polysensory area in superior temporal sulcus of the macaque. J Neurophysiol 46:369-384.

Buneo CA, Andersen RA (2006) The posterior parietal cortex: sensorimotor interface for the planning and online control of visually guided movements. Neuropsychologia 44:2594-2606.

Bursztyn LL, Ganesh G, Imamizu H, Kawato M, Flanagan JR (2006) Neural correlates of internal-model loading. Curr Biol 16:2440-2445.

Canavan AG, Passingham RE, Marsden CD, Quinn N, Wyke M, Polkey CE (1990) Prism adaptation and other tasks involving spatial abilities in patients with Parkinson's disease, patients with frontal lobe lesions and patients with unilateral temporal lobectomies. Neuropsychologia 28:969-984.

Clower DM, Hoffman JM, Votaw JR, Faber TL, Woods RP, Alexander GE (1996) Role of posterior parietal cortex in the recalibration of visually guided reaching. Nature 383:618-621.
Colent C, Pisella L, Bernieri C, Rode G, Rossetti Y (2000) Cognitive bias induced by visuo-motor adaptation to prisms: a simulation of unilateral neglect in normal individuals? Neuroreport 11:1899-1902.

Connolly JD, Andersen RA, Goodale MA (2003) FMRI evidence for a 'parietal reach region' in the human brain. Exp Brain Res 153:140-145.

Desmurget M, Gréa H, Grethe JS, Prablanc C, Alexander GE, Grafton ST (2001) Functional anatomy of nonvisual feedback loops during reaching: a positron emission tomography study. J Neurosci 21:2919-2928.

Diedrichsen J, Hashambhoy Y, Rane T, Shadmehr R (2005) Neural correlates of reach errors. J Neurosci 25:9919-9931.

Friston KJ, Ashburner J, Frith CD, Poline JB, Heather JD, Frackowiak RS (1995a) Spatial registration and normalisation of images. Hum Brain Mapp 2:165-189.

Friston KJ, Holmes AP, Worsley KJ, Poline JB, Frith CD, Frackowiak RS (1995b) Statistical parameter maps in functional imaging: a general linear approach. Hum Brain Mapp 2:189-210.

Girgenrath M, Bock O, Seitz RJ (2008) An fMRI study of brain activation in a visual adaptation task: activation limited to sensory guidance. Exp Brain Res 184:561-569.

Graydon FX, Friston KJ, Thomas CG, Brooks VB, Menon RS (2005) Learning-related fMRI activation associated with a rotational visuomotor transformation. Brain Res Cogn Brain Res 22:373-383.

Halligan PW, Fink GR, Marshall JC, Vallar G (2003) Spatial cognition: evidence from visual neglect. Trends Cogn Sci 7:125-133.

Imamizu H, Miyauchi S, Tamada T, Sasaki Y, Takino R, Pütz B, Yoshioka T, Kawato M (2000) Human cerebellar activity reflecting an acquired internal model of a new tool. Nature 403:192-195.

Inoue K, Kawashima R, Satoh K, Kinomura S, Goto R, Sugiura M, Ito M, Fukuda H (1997) Activity in the parietal area during visuomotor learning with optical rotation. Neuroreport 8:3979-3983.

Karnath HO, Perenin MT (2005) Cortical control of visually guided reaching: evidence from patients with optic ataxia. Cereb Cortex 15:1561-1569.

Karnath HO, Ferber S, Himmelbach M (2001) Spatial awareness is a function of the temporal not the posterior parietal lobe. Nature 411:950-953.

Karnath HO, Fruhmann BM, Küker W, Rorden C (2004) The anatomy of spatial neglect based on voxelwise statistical analysis: a study of $140 \mathrm{pa}-$ tients. Cereb Cortex 14:1164-1172.

Luauté J, Michel C, Rode G, Pisella L, Jacquin-Courtois S, Costes N, Cotton F, le Bars D, Boisson D, Halligan P, Rossetti Y (2006) Functional anatomy of the therapeutic effects of prism adaptation on left neglect. Neurology 66:1859-1867.

Martin TA, Keating JG, Goodkin HP, Bastian AJ, Thach WT (1996) Throwing while looking through prisms. I. Focal olivocerebellar lesions impair adaptation. Brain 119:1183-1198.

Medendorp WP, Goltz HC, Vilis T, Crawford JD (2003) Gaze-centered updating of visual space in human parietal cortex. J Neurosci 23:6209-6214.

Mesulam MM (1999) Spatial attention and neglect: parietal, frontal and cingulate contributions to the mental representation and attentional targeting of salient extrapersonal events. Philos Trans R Soc Lond B Biol Sci 354:1325-1346.

Miall RC, Imamizu H, Miyauchi S (2000) Activation of the cerebellum in co-ordinated eye and hand tracking movements: an fMRI study. Exp Brain Res 135:22-33.

Michel C (2006) Simulating unilateral neglect in normals: myth or reality? Restor Neurol Neurosci 24:419-430.

Michel C, Rossetti Y, Rode G, Tilikete C (2003) After-effects of visuomanual adaptation to prisms on body posture in normal subjects. Exp Brain Res 148:219-226.

Michel C, Pisella L, Prablanc C, Rode G, Rossetti Y (2007) Enhancing visuomotor adaptation by reducing error signals: single-step (aware) versus multiple-step (unaware) exposure to wedge prisms. J Cogn Neurosci 19:341-350.

Oldfield RC (1971) The assessment and analysis of handedness: the Edinburgh inventory. Neuropsychologia 9:97-113.

Pisella L, Michel C, Gréa H, Tilikete C, Vighetto A, Rossetti Y (2004) Preserved prism adaptation in bilateral optic ataxia: strategic versus adaptive reaction to prisms. Exp Brain Res 156:399-408.

Pisella L, Rossetti Y, Michel C, Rode G, Boisson D, Pélisson D, Tilikete C (2005) Ipsidirectional impairment of prism adaptation after unilateral lesion of anterior cerebellum. Neurology 65:150-152.

Pisella L, Binkofski F, Lasek K, Toni I, Rossetti Y (2006a) No doubledissociation between optic ataxia and visual agnosia: multiple sub- 
streams for multiple visuo-manual integrations. Neuropsychologia 44:2734-2748.

Pisella L, Rode G, Farnè A, Tilikete C, Rossetti Y (2006b) Prism adaptation in the rehabilitation of patients with visuo-spatial cognitive disorders. Curr Opin Neurol 19:534-542.

Ramnani N (2006) The primate cortico-cerebellar system: anatomy and function. Nat Rev Neurosci 7:511-522.

Redding GM, Wallace B (1996) Adaptive spatial alignment and strategic perceptual-motor control. J Exp Psychol Hum Percept Perform 22:379-394.

Redding GM, Rossetti Y, Wallace B (2005) Applications of prism adaptation: a tutorial in theory and method. Neurosci Biobehav Rev 29:431-444.

Rode G, Pisella L, Rossetti Y, Farnè A, Boisson D (2003) Bottom-up transfer of sensory-motor plasticity to recovery of spatial cognition: visuomotor adaptation and spatial neglect. Prog Brain Res 142:273-287.

Rossetti Y, Koga K, Mano T (1993) Prismatic displacement of vision induces transient changes in the timing of eye-hand coordination. Percept Psychophys 54:355-364.

Rossetti Y, Rode G, Pisella L, Farné A, Li L, Boisson D, Perenin MT (1998) Prism adaptation to a rightward optical deviation rehabilitates left hemispatial neglect. Nature 395:166-169.

Schmahmann JD, Doyon J, McDonald D, Holmes C, Lavoie K, Hurwitz AS, Kabani N, Toga A, Evans A, Petrides M (1999) Three-dimensional MRI atlas of the human cerebellum in proportional stereotaxic space. Neuroimage 10:233-260.

Seidler RD, Noll DC, Chintalapati P (2006) Bilateral basal ganglia activation associated with sensorimotor adaptation. Exp Brain Res 175:544-555.

Stein JF (1986) Role of the cerebellum in the visual guidance of movement. Nature 323:217-221.

Striemer C, Sablatnig J, Danckert J (2006) Differential influences of prism adaptation on reflexive and voluntary covert attention. J Int Neuropsychol Soc 12:337-349.

Striemer C, Blangero A, Rossetti Y, Boisson D, Rode G, Salemme R, Vighetto A, Pisella L, Danckert J (2008) Bilateral parietal lesions disrupt the beneficial effects of prism adaptation: evidence from a patient with optic ataxia. Exp Brain Res. Advance online publication. Retrieved December 23, 2008. doi:10.1007/S00221-008-1303-2.

Suchan B, Yágüez L, Wunderlich G, Canavan AG, Herzog H, Tellmann L, Hömberg V, Seitz RJ (2002) Hemispheric dissociation of visual-pattern processing and visual rotation. Behav Brain Res 136:533-544.

Weiner MJ, Hallett M, Funkenstein HH (1983) Adaptation to lateral displacement of vision in patients with lesions of the central nervous system. Neurology 33:766-772.

Zeffiro T (1995) Adaptation of visually-guided reaching to laterally displaced vision: a regional cerebral blood flow study [abstract]. Hum Brain Mapp Suppl 1:333. 